\title{
A Mote-in-the-Loop Approach for Exploring Communication Strategies for Sensor Networks
}

\author{
Minyan Hong ${ }^{1}$, Erik Björnemo ${ }^{1}$, Thiemo Voigt $^{2}$ \\ ${ }^{1}$ Uppsala University, Sweden \\ ${ }^{2}$ Swedish Institute of Computer Science (SICS), Kista, Sweden
}

\section{Introduction}

Sensor networks are being deployed in a range of different environments, such as industry plants, rainforests and offices. Each environment has its own characteristics and the appropriate communication strategy will differ accordingly - packet sizes, retransmission schemes, error correcting codes, etc. It is, however, difficult to investigate the most appropriate communication strategies for the environment of an intended deployment. On the one hand, simulations are seldom realistic enough as they do not model the environment in every intricate detail. On the other hand, real-world experiments with deployed nodes are important but time-consuming, difficult to repeat, and to some extent dependent on hardware and software. For example, a bug in the software might make measurements collected during an extensive time useless. We need an easier way of testing which still captures realistic communication environments and provides repeatability.

We propose a new approach to investigate communication strategies. Our approach uses a combination of on-site radio channel and interference measurements, real sensor network hardware as well as a signal analyser and a signal generator. The advantage of our approach is that once the channel measurements are made, we have a deterministic and repeatable way of investigating the most suitable communication strategy in the lab and for different sensor node hardware. Additionally, we can quickly test new hardware and new implementations by simply recording new packets.

\section{Approach}

The setup consists of two motes, a vector signal analyser (VSA) and a vector signal generator $(\mathrm{VSG})^{3}$, see Figure 2 . A modern vector signal analyser/generator is an advanced instrument with the following typical characteristics: Large frequency range; Large signal bandwidth; Accurate power reading/setting. These features render the instrument flexible and facilitate tests outside the reach of mote-to-mote communication such as the transmission of recorded signals at very precisely set power levels.

The motes are TmoteSky sensor nodes [3] which feature a CC2420 radio and run the Contiki operating system. The sending mote sends one or more packets that the signal analyser resolves to in-phase and quadrature (IQ) values that can be stored on

\footnotetext{
${ }^{3}$ In our case the 2810 VSA and the 2910 VSG from Keithley.
} 


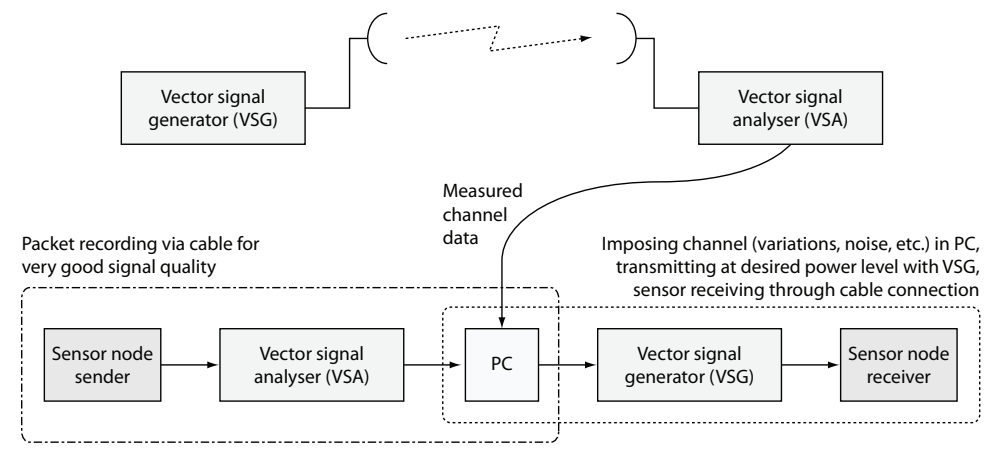

Fig. 1. Experimental setup for repeatable testing of communication strategies using real channel data.

the PC, for example, using Matlab. The radio communication between mote and signal analyser is via a cable to avoid external interference and achieve large signal-to-noise ratio $(>60 \mathrm{~dB})$. With software on the $\mathrm{PC}$, we can instruct the signal generator to replay the packets received by the signal analyser and transmit them to the mote as depicted in Figure 2. We can further vary the output power of the signal generator, e.g. according to measured channel gains. Moreover, we can modify the outgoing signals by e.g. adding measured or simulated interference and noise. In particular, we are able to collect measured channel data from different environments to emulate the impact of the environment on communation. This way, we expect to be able to find communcation strategies tailored to the environment. At the receiving mote we can measure e.g. packet reception rate but also retrieve the received signal strength indicator (RSSI), the link quality indicator (LQI) and noise floor values from the on-board radio.

\section{Evaluation and proof of concept}

\subsection{Basic RSSI experiment}

We verify the CC2420's RSSI readings by repeatedly replaying a recorded packet at increasing power levels. Figure 2 shows the results over the power range in which the motes actually receives the packets and can hence measure and report the RSSI (down to approximately $-95 \mathrm{dBm}$ ). The figure shows the expected overall linear relationship, with a small variance in the RSSI readings. However, we specifically note two regions - at output powers of $-40 \mathrm{dBm}$ and $-25 \mathrm{dBm}$ - where the linear relationship between RSSI and VSG output power is disturbed and the sample variance is larger. This reflects an inaccuracy of in the RSSI reading mechanism that also Chen and Terzis have observed [2]. Note that this inaccuracy thus confirms the correctness of our approach.

\subsection{Repeatable test of communication in fading channels}

While real world deployments in one respect constitute the ultimate test of a sensor network and its communication strategies, it can be very difficult to compare results for 


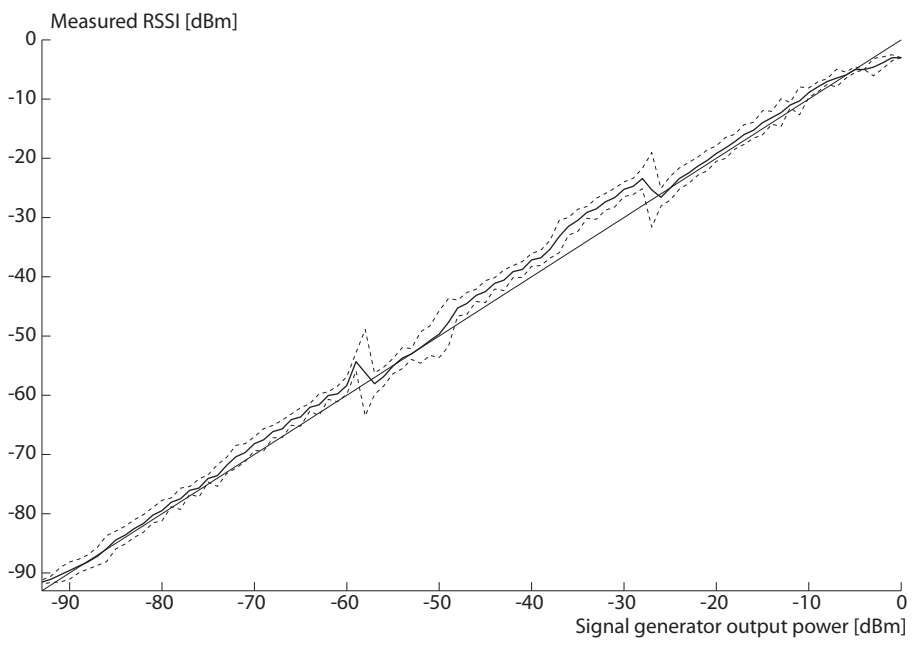

Fig. 2. RSSI from the CC 2420 as a function of the VSG signal output power. The mean \pm 3 standard deviations are given together with the ideal straight-line response. The curves are based on 10000 RSSI readings per power setting.

different deployments at different times. One reason is that variations in link quality - channel fading - are different at different times and locations. During research and development, it is therefore desirable to have a repeatable approach which still is much more realistic than simulation. Our proposed approach is a step in this direction, and we here show an example of the results we can achieve using real-world channel data. The channel characteristics used here consists of traces that we have collected in office and forest environments [1].

In Figure 3 the general procedure for including fading and interference is depicted. Note that there is a choice when it comes to the thermal noise as it can either be introduced artificially in the PC, as part of $n(\tau, t)$, or by using the real receiver noise and a scaled output power from the VSG. We used the latter approach and studied only the channel impact without interference for illustrative purposes. By the use of 10000 packets for each received average signal-to-noise ratio, we obtained packet error rate curves for three cases: No fading, measured office fading and measured forest fading. The channel data was applied so that block fading was achieved, that is a fairly constant channel during packet transmissions.

The results in Figure 4 show how the fading introduces error floors, starting at packet error rates of around 3 percent. The difference between the fading channels is not as extreme as one might expect, but it should be noted that the terms "line of sight" and "non line of sight" are inadequate to describe the difference. In fact, the office setting allowed some penetration through walls which resulted in "partial line of sight" (nonRayleigh fading). Additionally, the forest setting was not pure line of sight because of the antennas being very close to the ground. 


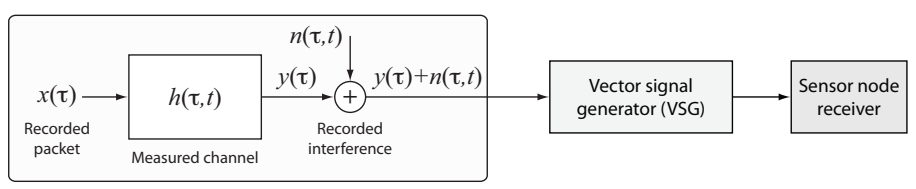

Fig. 3. Introduction of fading channel and interference. The packet $x(\tau)$, the channel $h(\tau, t)$ and the interference $n(\tau, t)$ are all complex-valued to contain both amplitude and phase information. The variable $t$ shows that the channel and the interference can have time-varying characteristics.

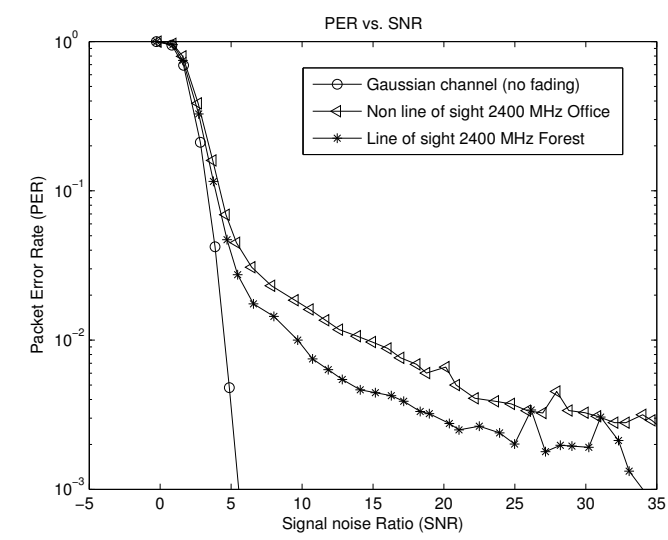

Fig. 4. Packet error rate (PER) for a range of signal-to-noise ratios (SNR). The channel was block fading, that is to say roughly constant during a packet but changing on an inter-packet time scale.

\section{Conclusions}

We have presented Mote-in-the-Loop, a new approach for communication strategy exploration. With some further extensions such as feedback from the sensor node to the VSG in order to trigger retransmission we believe that Mote-in-the-Loop will become a powerful and useful tool.

Acknowledgements. This work was funded by the Uppsala VINN Excellence Center for Wireless Sensor Networks WISENET, partly funded by VINNOVA.

\section{References}

1. E. Björnemo. Energy Constrained Wireless Sensor Networks : Communication Principles and Sensing Aspects. Institutionen för teknikvetenskaper, Uppsala University, 2009.

2. Y. Chen and A. Terzis. On the Mechanisms and Effects of Calibrating RSSI Measurements for 802.15. 4 Radios. In European Conference on Wireless Sensor Networks, Coimbra, Portugal, Feb. 2010.

3. J. Polastre, R. Szewczyk, and D. Culler. Telos: Enabling ultra-low power wireless research. In Proc. IPSN/SPOTS'05, Los Angeles, CA, USA, Apr. 2005. 